\title{
DETERMINAÇÃO DE MERCÚRIO EM FÍGADO DE TETRÁPODES MARINHOS POR ESPECTROMETRIA DE FLUORESCÊNCIA ATÔMICA ACOPLADA A GERAÇÃO DE VAPOR FRIO (CV-AFS) E ESPECTROMETRIA DE MASSA COM FONTE DE PLASMA INDUTIVAMENTE ACOPLADO (ICP-MS): UMA COMPARAÇÃO SISTEMÁTICA ENTRE AS DUAS TÉCNICAS
}

\author{
Raiza F. da Silva ${ }^{a}$, Siomara D. da Rocha ${ }^{\mathrm{b}, \mathrm{c}}$, Amauri A. Menegárioa,*,®, Jorge H. Pedrobom ${ }^{\mathrm{a}}$, Everton T. Sulato ${ }^{\mathrm{a}}$, Karen S. \\ Luko $^{a}$, Lucas Pellegrini Elias ${ }^{\text {a }, ~ L u c i a ~ M . ~ d e ~ S o u z a ~ O l i v e i r a ~}{ }^{a}$ e Ézio Sargentini Junior ${ }^{c}$ \\ ${ }^{a}$ Centro de Estudos Ambientais, Universidade Estadual Paulista, 13506-900 Rio Claro - SP, Brasil \\ bepartamento de Química, Universidade Federal do Amazonas, 69067-005 Manaus - AM, Brasil \\ 'Instituto Nacional de Pesquisas da Amazônia, 69060-001 Manaus - AM, Brasil
}

Recebido em 31/07/2020; aceito em 13/10/2020; publicado na web em 20/11/2020

\begin{abstract}
DETERMINATION OF MERCURY IN LIVER OF MARINE TETRAPODS BY COLD VAPOR ATOMIC FLUORESCENCE SPECTROMETRY AND INDUCTIVELY COUPLED PLASMA MASS SPECTROMETRY IN BIOLOGICAL SAMPLES: A SYSTEMATIC COMPARISON BETWEEN THE TWO TECHNIQUES. This study compares the techniques cold vapour atomic fluorescence spectrometry (CV-AFS) and inductively coupled plasma mass spectrometry (ICP-MS) for the determination of $\mathrm{Hg}$ in biological samples (hepatic tissue from marine tetrapods). Acid digestions were performed for the samples and aliquots of them were analysed by ICP-MS and CV-AFS. To minimize the Hg memory effect in its determination by ICP-MS, gold $\left(200 \mu \mathrm{g} \mathrm{L}^{-1}\right.$ in $5 \% \mathrm{v} \mathrm{v}^{-1} \mathrm{HCl}$ ) was added to the sample digests. The detection limits were $0.02 \mathrm{mg} \mathrm{kg}^{-1}$ for ICP-MS and $0.01 \mathrm{mg} \mathrm{kg}^{-1}$ for CV-AFS. Accuracy was assessed by the calculation of the recovery obtained from TORT-3 (lobster hepatopancreas) certified reference material analysis. Accurate results were obtained for both CV-AFS and ICP-MS. The comparison of the results obtained for hepatic tissue with and without lyophilization showed a significant difference among them (at 95\% of confidence level), evidencing losses of $\mathrm{Hg}$ in the lyophilization process.
\end{abstract}

Keywords: Mercury; Marine Tetrapod; Hg Memory Effect; ICP-MS; CV-AFS.

\section{INTRODUÇÃO}

O Hg, assim como os outros elementos estáveis constantes na tabela periódica, resiste aos processos de degradação da natureza e, como consequência, pode permanecer por vários anos no ambiente, passando por diversas transformações químicas e biológicas sem perder seu efeito tóxico. ${ }^{1}$ Devido a essas características, é um elemento que desperta grande interesse em estudos, principalmente à sua mobilidade no ambiente. Fenômenos naturais, como mudanças nas condições de temperatura, pressão atmosférica e ações antrópicas, como atividades industriais, podem modificar o ciclo natural do $\mathrm{Hg}$, elevando as concentrações deste elemento nos diversos compartimentos ambientais. Dessa forma, a cadeia alimentar é afetada nos diversos níveis tróficos, sendo possível a ocorrência de bioacumulação e biomagnificação, o que aumenta o risco desse elemento para a saúde humana e ambiental. ${ }^{2-4}$

Neste contexto a determinação de $\mathrm{Hg}$ é de fundamental importância para avaliar o impacto causado no meio ambiente. Portanto, o desenvolvimento de métodos para determinação de $\mathrm{Hg}$ em amostras biológicas é necessário, devido aos problemas relacionados à extração e preservação do analito na amostra e ao método de quantificação empregado..$^{5-7}$

A técnica de Espectrometria de Fluorescência Atômica acoplada à Geração de Vapor a Frio (CV-AFS) é considerada uma das mais sensíveis para determinação de $\mathrm{Hg}$ em diversos tipos de amostras, tais como ambientais, biológicas e alimentares, por ser pouco susceptível à interferência de matriz, ser de baixo custo operacional, permitir limite de detecção excelente (ao nível de ng $\mathrm{L}^{-1}$ ). ${ }^{8,9}$ A determinação de $\mathrm{Hg}$ requer um limite de detecção baixo e a espectrometria de

*e-mail: amauri.antonio-menegario@unesp.br massa com fonte de plasma indutivamente acoplado (ICP-MS), em princípio, também pode ser utilizada. No entanto, na determinação desse elemento através da ICP-MS pode ocorrer efeito memória. ${ }^{10,11}$ Lamble e Hill ${ }^{12}$ propuseram um método para a determinação de $\mathrm{Hg}$ total por CV-AFS baseado em digestão on-line de amostras ambientais assistida por micro-ondas. As amostras, na forma de suspensão, foram injetadas em carregador (ácido clorídrico e soluções de brometo e bromato) para uma bobina de reação posicionada dentro de um forno micro-ondas. Foi reportado um limite de detecção (LD) de $0,013 \mu \mathrm{g} \mathrm{L}^{-1}$. Os resultados obtidos foram concordantes com os valores certificados para $\mathrm{Hg}$ em sedimento e músculo de cação marinhos (PACS-1 e DORM-2).

Tao et al. ${ }^{13,14}$ reportaram um método simples e rápido, sem a necessidade de uma técnica cromatográfica, para a determinação de Hg inorgânico e total em amostras de peixes, utilizando a técnica de Espectrometria de Absorção Atômica com geração química de vapor frio (CV-AAS). As amostras foram solubilizadas usando hidróxido de tetrametilamônio (TMAH). O Hg inorgânico foi liberado pela injeção em fluxo de L-cisteína e depois reduzido para $\mathrm{Hg}$ metálico com $\mathrm{SnCl}_{2}$. O limite de detecção para o mercúrio inorgânico foi de $0,1 \mu \mathrm{g} \mathrm{L}^{-1} \mathrm{e}$ a precisão da determinação foi caracterizada por um desvio-padrão relativo de $2 \%$ para uma solução de $20 \mu \mathrm{g} \mathrm{L}^{-1}$. O método proposto foi validado pela análise de um conjunto de materiais biológicos marinhos de referência, DORM-2 (músculo de cação), DOLT-2 (fígado de cação) e TORT-2 (hepatopâncreas de lagosta).

Park e $\mathrm{Do}^{15}$ também analisaram $\mathrm{Hg}$ inorgânico e total em amostras de material biológico marinho $(0,1 \mathrm{~g})$, por meio de diluição isotópica (com ${ }^{199} \mathrm{Hg}$ ) por ICP-MS acoplado a geração de vapor frio (CV-ICP-MS) após digestão com hidróxido de tetrametilamonio (TMAH). $\mathrm{O}$ agente redutor $\mathrm{SnCl}_{2}$ foi utilizado para determinações de $\mathrm{Hg}$ inorgânico e, para $\mathrm{Hg}$ total, $\mathrm{NaBH}_{4}$. $\mathrm{O}$ método proposto foi 
validado a partir da análise de três materiais certificados: músculo de cação DORM-2, de ostra NIST SRM1566b e de atum CRM KRISS (numeração ainda não atribuída no período da publicação do estudo). O limite de detecção do método encontrado foi de $0,018 \mu \mathrm{g} \mathrm{kg}^{-1}$.

Barcelos ${ }^{16}$ reportou a determinação de $\mathrm{Hg}$ em amostra de tecido de peixe, utilizando $\mathrm{CV}$-AFS. As amostras foram digeridas em forno micro-ondas $\left(0,5 \mathrm{~g}\right.$ de amostra e adição de $6 \mathrm{~mL}$ de $\mathrm{HNO}_{3} 65 \% \mathrm{v} \mathrm{v}^{-1}$ e $1 \mathrm{~mL}$ de $\mathrm{H}_{2} \mathrm{O}_{2} 30 \% \mathrm{~m} \mathrm{~m}^{-1}$ ). O LD do método foi $0,13 \mu \mathrm{g} \mathrm{kg}^{-1}$ e a precisão caracterizada por um desvio-padrão relativo de $1,8 \%$.

ICP-MS é uma técnica de elevado custo capital e operacional. Para a determinação de $\mathrm{Hg}$ por meio desta técnica, podem ocorrer interferências não espectrais e, menos corriqueiramente, interferências espectrais. ${ }^{16}$ A técnica é caracterizada pela sua alta sensibilidade, com baixos LDs, além de outras vantagens como a determinação multielementar. ${ }^{13,17-20}$

Para minimizar o efeito memória do $\mathrm{Hg}$ em suas determinações por ICP-MS, pode-se adicionar Au ou 2-mercaptoethanol na solução da amostra. ${ }^{2}$ Harrington et al. ${ }^{21}$ analisaram amostras de tecido de peixe, que foram digeridas em forno micro-ondas, mediante combinação de sistema de injeção em fluxo com o sistema de introdução de amostras do ICP-MS, utilizando 2-mercaptoethanol - 0,05\% ( $\left.\mathrm{v} \mathrm{v}^{-1}\right)$ como solução carregadora. Com essa abordagem, o LD foi $5,1 \mu \mathrm{g} \mathrm{L} \mathrm{L}^{-1}$. Zhu e Alexandratos ${ }^{22}$ minimizaram o efeito memória de $\mathrm{Hg}$ em determinações por espectrometria de emissão óptica com plasma indutivamente acoplado com a adição de agentes complexantes (tioureia e cloreto de ouro). Segundo os autores, a utilização de $\mathrm{Au}$ (III) resulta em melhores resultados para o desaparecimento mais rápido do efeito de memória.

Gelaude et al. ${ }^{23}$ utilizaram a vaporização eletrotérmica acoplada a ICP-MS (SS-ETV-ICPMS) para a determinação simultânea de metilmercúrio e $\mathrm{Hg}$ inorgânico em amostras biológicas (hepatopâncreas de lagosta e músculo de cação). Para quantificação, os autores utilizaram diluição isotópica, com espécie específica (enriquecido em ${ }^{200} \mathrm{Hg}$ ). Embora esse método tenha a vantagem de não requerer prévia decomposição da amostra, evitando problemas de contaminação e perda do analito, é difícil de ser implementado devido à instrumentação complexa.

Um método baseado no acoplamento CV-ICP-MS foi desenvolvido por Kenduzler et al. ${ }^{24}$ para a determinação de $\mathrm{Hg}$ (II) e $\mathrm{Hg}$ total em otólitos de peixes. $\mathrm{O} \mathrm{NaBH}$ foi usado como agente redutor e a solução de ferrocianeto de potássio foi utilizada para a eliminação do efeito memória. Os autores reportaram limites de detecção de 4,2 e 6,4 ng L $\mathrm{L}^{-1}$ para $\mathrm{Hg}$ (II) e $\mathrm{Hg}$ total, respectivamente.

Ainda na etapa de tratamento e processamento das amostras, a influência da liofilização na integridade das espécies de $\mathrm{Hg}$ é pouco reportada na literatura. Amostras frescas são geralmente armazenadas, congeladas e posteriormente liofilizadas. A liofilização é frequentemente utilizada com a finalidade de pré-concentrar o analito por secagem e melhorar a preservação das amostras. ${ }^{25}$ Resultados obtidos a partir deste procedimento foram reportados, tendo sido similares aos obtidos a partir de métodos convencionais de secagem (para temperaturas abaixo de $100{ }^{\circ} \mathrm{C}$ ) ${ }^{26} \mathrm{Contudo}$, a utilização da liofilização deve ser avaliada com cautela, pois este procedimento pode resultar em perdas de $\mathrm{Hg}^{27}$

Para assegurar a qualidade da análise, parâmetros importantes, tais como sensibilidade, possíveis interferentes e efeito memória devem ser examinados. ${ }^{28,29}$ Nesse trabalho, fez-se uma comparação sistemática entre as duas técnicas analíticas (ICP-MS e CV-AFS) que podem ser utilizadas para a determinação de $\mathrm{Hg}$ total em amostras de fígado de tetrápodes marinhos. Avaliou-se ainda o efeito memória do $\mathrm{Hg}$ em ICP-MS e também a potencial perda do analito durante o processo de liofilização de amostras de tetrápodes marinhos.

\section{PARTE EXPERIMENTAL}

\section{Equipamentos e acessórios}

As amostras foram analisadas por ICP-MS (modelo $X$ series 2-Thermo Scientific) e por CV-AFS (modelo Millennium SystemPS Analytical), ambos os equipamentos operados de acordo com as especificações constantes na Tabela 1. Os demais equipamentos e acessórios utilizados foram: balança analítica (AB204 - Mettler Toledo, EUA); pipetas automáticas (Gilson, França; Boeco, Alemanha); digestor de amostras por micro-ondas (Speedwave four SW4 - Berghof - Alemanha) e vidrarias de uso rotineiro em laboratório de análises químicas.

Tabela 1. Parâmetros de operação dos equipamentos de ICP-MS e CV-AFS

\begin{tabular}{ccc}
\hline Parâmetros & Condição de operação \\
\hline Potência da radiofrequência & $1349 \mathrm{~W}$ \\
Vazão do gás de nebulização & $0,82 \mathrm{~L} \mathrm{~min}^{-1}$ \\
Vazão do gás auxiliar & $0,80 \mathrm{~L} \mathrm{~min}^{-1}$ \\
Vazão do gás do plasma & $13,0 \mathrm{~L} \mathrm{~min}^{-1}$ \\
Vazão da amostra & $1,5 \mathrm{~mL} \mathrm{~min}^{-1}$ \\
Padrões internos & Bi, Tb \\
ICP-MS Nebulizador & Burgener, Mira mist \\
& Cone de amostragem & Níquel \\
Modo de leitura & Peak Jumping \\
Tempo total de aquisição & $6 \mathrm{~s}$ \\
$m / z$ & 202 \\
\hline Vazão da amostra & $5 \mathrm{~mL} \mathrm{~min}^{-1}$ \\
Vazão da solução de SnCl & $2,7 \mathrm{~mL} \mathrm{~min}^{-1}$ \\
Vazão do gás de arraste & $250 \mathrm{~mL} \mathrm{~min}^{-1}$ \\
Ganho & 100 \\
Tempo de espera & $15 \mathrm{~s}$ \\
Tempo total de aquisição & $30 \mathrm{~s}$ \\
Tempo de limpeza & $20 \mathrm{~s}$ \\
\hline
\end{tabular}

\section{Amostras}

Todas as amostras analisadas foram de tecido hepático de tetrápodes marinhos em estágio 2 de decomposição (por morte natural), encontrados em regiões costeiras do Brasil e, posteriormente, preservadas a $-80^{\circ} \mathrm{C}$ em ultrafreezer. As coletas foram realizadas pela Petrobrás e autorizadas pelo Instituto Brasileiro do Meio Ambiente e dos Recursos Naturais Renováveis (IBAMA).

Para comparar os resultados obtidos por ICP-MS e CV-AFS, analisaram-se 20 amostras in natura, sendo 14 de Chelonia mydas (tartaruga-verde), 4 de Larus dominicanus (gaivotão), 1 de Sotalia guianensis (boto-cinza), e 1 de Spheniscus magellanicus (pinguim-de-magalhães).

Outras 15 amostras, divididas em duas frações, in natura e com prévia liofilização foram analisadas por CV-AFS, sendo 9 Chelonia mydas, 4 Sotalia guianensis e 2 Sula leucogaster (atobá-marrom), e os resultados utilizados para comparação das análises das amostras in natura e liofilizadas.

Para avaliação da exatidão dos métodos foram analisadas uma amostra de referência (fígado bovino) e uma amostra de material de referência certificado (TORT-3, produzido pelo National Research Council of Canadá). 


\section{Reagentes e soluções}

Foram utilizados reagentes de grau analítico e água ultrapura do sistema de purificação Milli-Q Direct-Q ${ }^{\circledR} 5 \mathrm{UV}$, com resistividade de $18,2 \mathrm{M} \Omega \mathrm{cm}^{-1}$ para o preparo das amostras, padrões e soluções. Os ácidos $\mathrm{HNO}_{3}$ e $\mathrm{HCl}$ foram previamente purificados pelo processo de destilação abaixo do ponto de ebulição, duas vezes (bidestilados), para evitar contaminação por $\mathrm{Hg}$. Todos os materiais utilizados foram previamente descontaminados por imersão em $\mathrm{HNO}_{3} \quad 10 \%\left(\mathrm{v} \mathrm{v}^{-1}\right)$ durante quatro horas, em seguida lavados cinco vezes com água ultrapura e secos em capela de fluxo laminar antes do uso.

Foram preparadas soluções de calibração com concentrações de 0,$0 ; 0,1 ; 0,25 ; 0,5 ; 1,0$ e $2,0 \mu \mathrm{g} \mathrm{L}^{-1} \mathrm{de} \mathrm{Hg}$, obtidas a partir de diluições da solução padrão estoque certificada G1467V - 100 mg L-1 (SpecSol, Brasil), para a construção das curvas de calibração para ambas as técnicas CV-AFS e ICP-MS.

Para as determinações por CV-AFS, as amostras e as soluções de calibração foram preparadas em meio $\operatorname{KBr}\left(1,19 \% \mathrm{~m} \mathrm{v}^{-1}\right)$ / $\mathrm{KBrO}_{3}\left(0,28 \% \mathrm{~m} \mathrm{v}^{-1}\right), \mathrm{HCl}$ bidestilado (concentrado) e cloridrato de hidroxilamina $\left(5 \% \mathrm{~m} \mathrm{v}^{-1}\right)$. O íon $\mathrm{Hg}$ (II) foi reduzido a $\mathrm{Hg}^{0}$ com o uso do agente redutor $\mathrm{SnCl}_{2}$ em solução $2 \%\left(\mathrm{~m} \mathrm{v}^{-1}\right)$, segundo os métodos EPA 245.7 (águas) e EPA 7474 (sedimentos e tecidos).

Para as determinações por ICP-MS, as amostras e as soluções de calibração foram preparadas em $\mathrm{HCl}\left(5 \%, \mathrm{v} \mathrm{v}^{-1}\right)$ e $\mathrm{Au}\left(200 \mu \mathrm{g} \mathrm{L}^{-1}\right)$.

\section{Comparação entre determinações por CV-AFS e ICP-MS}

\section{Pré-tratamento de liofilização}

Para o processamento das amostras submetidas ao tratamento de liofilização, foi pesada uma alíquota de aproximadamente $5 \mathrm{~g}$ para cada amostra congelada $\left(-80^{\circ} \mathrm{C}\right)$. As alíquotas foram inseridas no liofilizador (Alpha 1-2 LDplus CHRIST, Holanda) sob as condições $-40{ }^{\circ} \mathrm{C}$ e 0,11 mbar por 72 horas. Finalizado o procedimento de liofilização, as amostras foram maceradas e homogeneizadas para posteriormente serem digeridas.

\section{Digestão das amostras}

Uma massa de $1 \mathrm{~g}$ de cada amostra in natura e $0,125 \mathrm{~g}$ de cada alíquota liofilizada foi pesada em frascos para digestão em forno micro-ondas fornecidos pelo fabricante, adicionados $2 \mathrm{~mL}$ de $\mathrm{HNO}_{3}$ concentrado $\left(65 \% \mathrm{v} \mathrm{v}^{-1}\right), 6 \mathrm{~mL}$ de $\mathrm{HCl}$ concentrado $\left(37 \% \mathrm{v} \mathrm{v}^{-1}\right)$, e deixada em repouso por uma noite em temperatura ambiente para uma digestão prévia. Em seguida, os frascos foram inseridos no rotor e transferidos para o forno micro-ondas, para a digestão final das amostras. Ao final do procedimento, o extrato foi transferido quantitativamente para tubo centrífuga por meio da lavagem do conteúdo com água ultrapura, no mínimo três vezes, sendo avolumado para o volume final de $15 \mathrm{~mL}$. Para cada amostra, foi realizado um procedimento de digestão.

\section{Determinações por ICP-MS}

Uma alíquota de $0,3 \mathrm{~mL}$ do digerido de cada amostra foi transferida para tubo centrífuga de $15 \mathrm{~mL}$ e adicionado $0,03 \mathrm{~mL}$ da solução de $\mathrm{Au}, 0,75 \mathrm{~mL}$ de $\mathrm{HCl}$ concentrado e o volume completado com água ultrapura. Três réplicas de diluições foram preparadas e analisadas por ICP-MS.

\section{Determinações por $C V$-AFS}

Uma segunda alíquota de $1 \mathrm{~mL}$ do digerido de cada amostra foi transferida para tubos centrífuga de $50 \mathrm{~mL}$ e adicionados $2 \mathrm{~mL}$ de uma solução contendo $\mathrm{KBr}\left(1,19 \% \mathrm{~m} \mathrm{v}^{-1}\right)$ e $\mathrm{KBrO}_{3}\left(0,28 \% \mathrm{~m} \mathrm{v}^{-1}\right)$, previamente preparada. Em seguida, foram adicionados $2,5 \mathrm{~mL}$ de $\mathrm{HCl}$ concentrado e o volume elevado para $50 \mathrm{~mL}$ com água ultrapura.
Três réplicas de diluições foram preparadas e analisadas por CV-AFS.

\section{Comparação entre amostras in natura e liofilizada}

Com o intuito de comparar os resultados obtidos entre as amostras in natura (em base úmida, $\mathrm{mg} \mathrm{L}^{-1}$ ) e liofilizadas, o teor de umidade de cada amostra foi estabelecido através da diferença de massa antes e depois do procedimento de liofilizaçao. Dessa forma, o teor de umidade de cada amostra foi utilizado para converter a concentração de $\mathrm{Hg}$ para base seca $\left(\mathrm{mg} \mathrm{kg}^{-1}\right)$ por meio da equação 1:

$$
\mathrm{C}_{\text {seca }}=\mathrm{C}_{\text {úmida }} /\left[\left(100-\mathrm{Um}_{\text {amostras }}\right) / 100\right]
$$

em que: $\mathrm{C}_{\text {seca }}=$ concentração em base seca $\left(\mathrm{mg} \mathrm{kg}^{-1}\right) ; \mathrm{C}_{\text {úmida }}=$ concentração em base úmida $\left(\mathrm{mg} \mathrm{L}^{-1}\right) ; \mathrm{Um}_{\text {amostras }}=$ umidade das amostras $(\%)$.

\section{Limites de detecção e limites de quantificação}

Os limites de detecção (LD) e quantificação (LQ) foram calculados mediante as equações 2 e $3 .^{30} \mathrm{O}$ desvio-padrão foi calculado com base em 9 determinações consecutivas do analito no branco analítico. ${ }^{31}$

$$
\begin{gathered}
\mathrm{LD}=t \times(s) \\
\mathrm{LQ}=10 \times(s)
\end{gathered}
$$

em que: $t=t$ (Student) tabelado, valor de 3,355 (nível de confiança de $99,5 \%) ; s=$ desvio padrão das 9 determinações consecutivas do analito (em $\mu \mathrm{g} \mathrm{L}^{-1}$ ou $\mathrm{mg} \mathrm{kg}^{-1}$ ).

\section{RESULTADOS E DISCUSSÃO}

Inicialmente foram realizadas análises das amostras de fígado bovino in natura (adicionada concentração conhecida de $\mathrm{Hg}$, i.e., spike) e do material de referência certificado (TORT-3) por CV-AFS. Os resultados obtidos foram bastante satisfatórios, com recuperações entre 93 e $96 \%$ para a amostra de fígado bovino e $107 \pm 13 \%$ para a amostra certificada.

\section{Avaliação do Efeito Memória de Hg}

Inicialmente foram realizados testes com soluções de $\mathrm{Hg}$ contendo $200 \mu \mathrm{g} \mathrm{L}{ }^{-1} \mathrm{Au}$ (III) para diferentes intervalos de concentração de $\mathrm{Hg}$ (cada intervalo representou uma curva de calibração), a fim de avaliar a eficiência do Au na diminuição do efeito memória nas determinações e estabelecer em que faixa de concentração de $\mathrm{Hg}$ esse método é eficiente. A primeira curva foi construída com soluções de calibração contendo $\mathrm{Hg}$ entre 0-2 $\mu \mathrm{g} \mathrm{L}^{-1}$, com $10 \mathrm{~s}$ para limpeza do sistema de introdução de amostra no ICP-MS $\left(\mathrm{HNO}_{3} 2 \% \mathrm{v} \mathrm{v}^{-1}\right)$, entre uma solução e outra; a segunda com soluções contendo concentrações entre 0-3 $\mu \mathrm{g} \mathrm{L}^{-1} \mathrm{de} \mathrm{Hg}$, também usando um tempo de limpeza de $10 \mathrm{~s} \mathrm{e}$, por fim, a terceira foi construída com soluções contendo concentrações entre 0-5 $\mu \mathrm{g} \mathrm{L}^{-1}$, com tempo de limpeza de $15 \mathrm{~s}$. Após a injeção de todas as soluções de calibração, ao término da análise da solução de maior concentração para cada curva, foi analisado novamente o branco da curva de calibração $\left(0 \mu \mathrm{g} \mathrm{L}^{-1}\right)$ repetidas vezes, para avaliar a limpeza do sistema.

Por meio da Figura 1 pode-se observar o efeito memória do Hg após a construção da curva de calibração, com soluções entre 0-5 $\mu \mathrm{g} \mathrm{L}^{-1}$, caracterizado pelo aumento da concentração de $\mathrm{Hg}$ determinada no branco. Para as outras duas curvas $\left(0-2 \mu \mathrm{g} \mathrm{L}^{-1} \mathrm{e}\right.$ 0 - $3 \mu \mathrm{g} \mathrm{L}^{-1}$ ), não há evidências de efeito memória. 

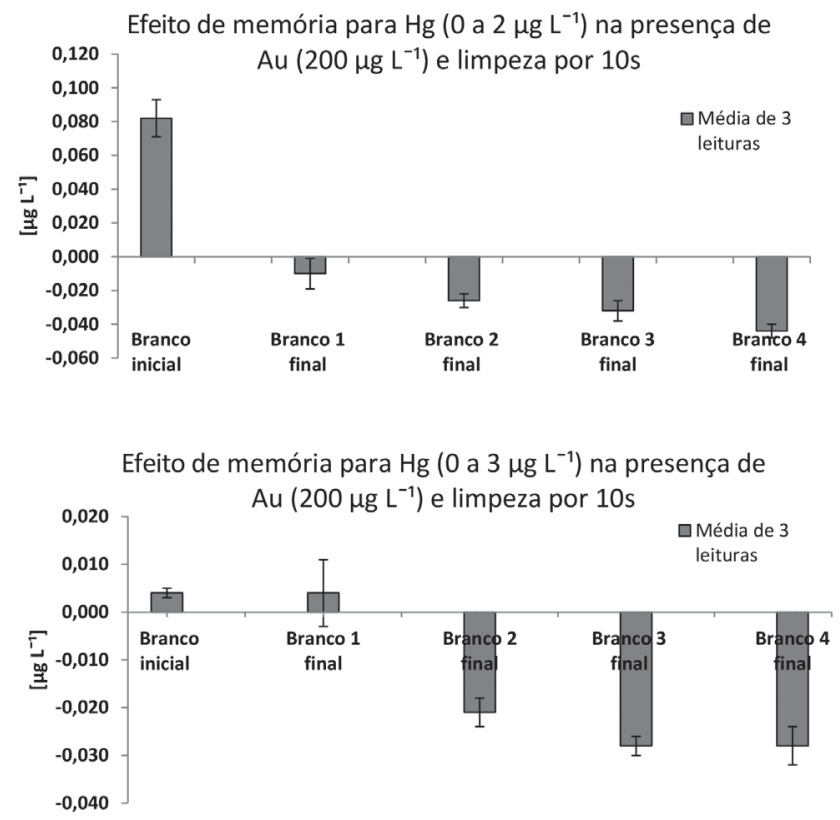

Efeito de memória para $\mathrm{Hg}\left(0\right.$ a $\left.5 \mu \mathrm{L} \mathrm{L}^{-1}\right)$ na presença de Au $\left(200 \mu \mathrm{g} \mathrm{L}^{-1}\right)$ e limpeza por $15 \mathrm{~s}$

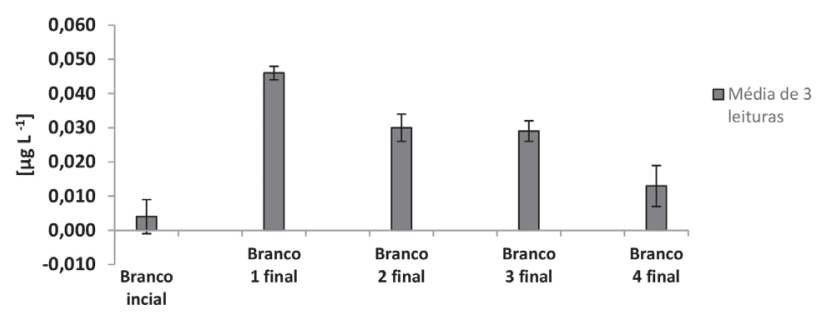

Figura 1. Avaliação do efeito memória do Hg em ICP-MS; branco inicial: concentração de Hg no branco da curva de calibração antes da análise das soluções de calibração; branco final: concentração de $\mathrm{Hg}$ no mesmo branco após a análise das soluções de calibração

$\mathrm{Chen}^{32}$ também avaliou o efeito de memória do $\mathrm{Hg}$, lavando com $\mathrm{HNO}_{3} 2 \%$ o sistema de introdução de amostras e com $200 \mu \mathrm{g} \mathrm{L}^{-1}$ de $\mathrm{Au}$ entre as amostras (curva analítica de 0 - $1 \mu \mathrm{g} \mathrm{L}^{-1}$ ). A concentração de $\mathrm{Hg}$ retornou ao valor inicial em cerca de $60 \mathrm{~s}$, tempo bastante superior ao observado no presente trabalho, em que soluções de calibração com concentração maior (até $3 \mu \mathrm{g} \mathrm{L}^{-1}$ ) não causaram o efeito memória após $10 \mathrm{~s}$ de lavagem do sistema.

\section{Limites de detecção}

Para os métodos do ICP-MS e CV-AFS, foi calculado o LD instrumental considerando-se o branco da curva de calibração. Para o cálculo, foi utilizada a equação 2 , obtendo-se $0,009,0,010$ e $0,011 \mu \mathrm{g} \mathrm{L}^{-1}$ para as curvas $0-2 \mu \mathrm{g} \mathrm{L}^{-1}, 0-3 \mu \mathrm{g} \mathrm{L}^{-1}$ e $0-5 \mu \mathrm{g} \mathrm{L}^{-1}$, respectivamente. Atribuímos o discreto aumento no valor do LD, para curvas de calibração com maior faixa de concentração, ao ajuste de pontos de cada curva.

Harrington et $a l .{ }^{21}$, em sua metodologia para reduzir o efeito memória na determinação de $\mathrm{Hg}$ por ICP-MS (solução carregadora contendo 2-mercaptoethanol, com injeção em fluxo), reportaram um valor de $\mathrm{LD}$ de $5,1 \mu \mathrm{g} \mathrm{L}^{-1}$, muito superior ao obtido neste trabalho (entre 0,009 e $0,011 \mu \mathrm{g} \mathrm{L}^{-1}$ ). Em outro estudo, Allibone et al., ${ }^{33}$ desenvolveram um método para a determinação rotineira de $\mathrm{Hg}$ em amostras de água potável por ICP-MS. Com a finalidade de minimizar o efeito de memória e preservar o Hg na solução, foram adicionados, em modo off-line $5 \mathrm{mg} \mathrm{L}^{-1}$ de $\mathrm{Au}$ e $\mathrm{HNO}_{3} 2 \%$ às amostras, soluções de calibração e água de lavagem do sistema de introdução das mesmas no ICP-MS. A adição de Au à água de lavagem evitou a retenção de $\mathrm{Hg}$ no sistema. Os autores reportaram um LD de 0,032 $\mu \mathrm{g} \mathrm{L} \mathrm{L}^{-1}$, também superior aos obtidos neste trabalho.

A Tabela 2 apresenta os valores de LD e LQ do presente método para ICP-MS e CV-AFS calculados a partir do branco analítico - vide item Digestão das amostras -.

Tabela 2. Limite de detecção (LD) e limite de quantificação (LQ) do método para ambas as técnicas

\begin{tabular}{ccc}
\hline Parâmetros & ICP-MS $\left(\mathrm{mg} \mathrm{kg}^{-1}\right)$ & CV-AFS $\left(\mathrm{mg} \mathrm{kg}^{-1}\right)$ \\
\hline LD & 0,02 & 0,01 \\
LQ & 0,06 & 0,03 \\
\hline
\end{tabular}

A análise estatística para comparação entre as duas técnica analíticas mostra que os valores médios de LD e LQ não foram significativamente diferentes $\left(\mathrm{t}_{\text {calculado }}=0,2952, \mathrm{t}_{\text {tabelado }}=2, \mathrm{n}=2, t\right.$ de Student pareado).

\section{Comparação dos resultados: ICP-MS e CV-AFS}

Os resultados das análises por CV-AFS e ICP-MS são apresentados na Tabela 3. Foi realizado o teste $F$ para verificar se os resultados poderiam ser considerados comparáveis. O teste $F$ indicou que os resultados não são significativamente diferentes entre $\mathrm{si}$, os dois conjuntos de dados foram avaliados mediante o teste $t$ de Student pareado, para o conjunto de dados obtidos para as amostras

Tabela 3. Concentração de $\mathrm{Hg}$ (em base úmida) nas amostras de fígado de tetrápodes marinhos in natura analisadas por ICP-MS e CV-AFS

\begin{tabular}{|c|c|c|}
\hline \multirow[t]{2}{*}{ Amostra } & ICP-MS & CV-AFS \\
\hline & $\begin{array}{l}\text { Média } \pm \text { desvio padrão } \\
\left(\mathrm{mg} \mathrm{kg}^{-1}\right)\end{array}$ & $\begin{array}{c}\text { Média } \pm \text { desvio padrão } \\
\left(\mathrm{mg} \mathrm{kg}^{-1}\right)\end{array}$ \\
\hline PT 472 & $0,150 \pm 0,021$ & $0,147 \pm 0,005$ \\
\hline PT 473 & $1,225 \pm 0,015$ & $1,189 \pm 0,006$ \\
\hline PT 474 & $0,212 \pm 0,030$ & $0,247 \pm 0,005$ \\
\hline PT 475 & $0,119 \pm 0,020$ & $0,123 \pm 0,006$ \\
\hline PT 476 & $0,624 \pm 0,054$ & $0,903 \pm 0,030$ \\
\hline PT 477 & $0,145 \pm 0,013$ & $0,143 \pm 0,020$ \\
\hline PT 478 & $0,104 \pm 0,041$ & $0,121 \pm 0,001$ \\
\hline PT 479 & $0,087 \pm 0,034$ & $0,100 \pm 0,004$ \\
\hline PT 480 & $0,364 \pm 0,009$ & $0,474 \pm 0,010$ \\
\hline PT 481 & $0,381 \pm 0,012$ & $0,335 \pm 0,007$ \\
\hline PT 482 & $1,225 \pm 0,015$ & $1,189 \pm 0,006$ \\
\hline PT 483 & $0,260 \pm 0,024$ & $0,212 \pm 0,007$ \\
\hline PT 484 & $0,220 \pm 0,049$ & $0,107 \pm 0,013$ \\
\hline PT 487 & $1,429 \pm 0,124$ & $1,429 \pm 0,022$ \\
\hline PT 488 & $1,624 \pm 0,055$ & $1,675 \pm 0,024$ \\
\hline PT 491 & $0,080 \pm 0,010$ & $0,043 \pm 0,002$ \\
\hline PT 493 & $1,648 \pm 0,014$ & $1,559 \pm 0,008$ \\
\hline PT 494 & $0,215 \pm 0,018$ & $0,158 \pm 0,004$ \\
\hline PT 495 & $0,128 \pm 0,010$ & $0,108 \pm 0,007$ \\
\hline PT 496 & $1,015 \pm 0,050$ & $0,929 \pm 0,007$ \\
\hline
\end{tabular}

Média e desvio padrão calculados para 3 leituras. 
in natura. $\mathrm{O}$ valor de $t$ calculado $(0,1703)$ foi menor que o tabelado $(2,09 ; n=20)$, indicando que não houve diferença significativa (com $95 \%$ de confiança) entre os resultados.

Visando comparar melhor os dados obtidos para as amostras in natura, esses também foram avaliados mediante correlação linear, conforme mostrado na Figura 2. O erro sistemático foi $<1 \%$ e a correlação boa, com $\mathrm{R}^{2}=0,9781(\mathrm{y}=0,9915 \mathrm{x}+0,0016)$, confirmado os resultados obtidos mediante o teste $t$. Assim sendo, ICP-MS pode ser uma alternativa para determinação de $\mathrm{Hg}$, possibilitando uma análise mais rápida que a CV-AFS.

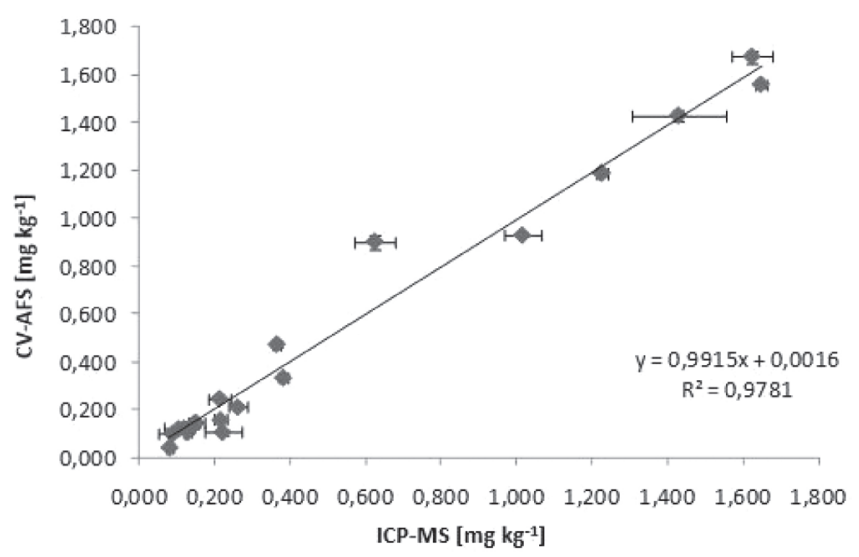

Figura 2. Correlação entre os valores de concentração de Hg encontrados em amostra de fígado de tetrápodes marinhos in natura por espectrometria de massa com fonte de plasma indutivamente acoplado (ICP-MS) e espectrometria de fluorescência atômica acoplada a geração de vapor frio (CV-AFS)

\section{Comparação dos resultados para amostras liofilizadas e amostras in natura}

Para avaliar a perda de $\mathrm{Hg}$ durante o processo de liofilização, foram analisadas amostras in natura e liofilizadas por CV-AFS. Os resultados das análises são apresentados na Tabela 4. Para efeito de comparação entre as amostras, os resultados foram normalizados em base seca (equação 1) e posteriormente foi aplicado o teste t de Student pareado. O valor calculado de $t$ foi $-4,3274$ e indicou que houve diferença significativa (ao nível de confiança de 95\%) entre os resultados, sugerindo que há perda de $\mathrm{Hg}$ durante o processo de liofilização.

A Figura 3 exibe a correlação dos valores de concentração de $\mathrm{Hg}$ em amostras in natura e liofilizadas analisadas por CV-AFS, nas quais foram observados erros sistemáticos (cerca de 10\%) para os dois conjuntos de dados $(y=1,1093 x+0,3165)$, conforme evidenciado pelo valor de inclinação da reta nesse conjunto, confirmando perda de $\mathrm{Hg}$ durante o processo de liofilização.

\section{CONCLUSÕES}

As análises mostraram que a determinação de $\mathrm{Hg}$ em tecido hepático de tetrápodes marinhos é possível por ICP-MS desde que sejam seguidas as condições de contorno para minimizar o efeito memória do $\mathrm{Hg}$ (concentração de $\mathrm{Hg}$ até $3 \mu \mathrm{g} \mathrm{L}^{-1}$ ). Os limites de detecção obtidos foram semelhantes para métodos baseados em ICP-MS e CV-AFS.

A avaliação da perda de $\mathrm{Hg}$ durante o processo de liofilização mostrou diferença significativa (nível de confiança de 95\%), evidenciando perda de $\mathrm{Hg}$, em média, de $10 \%$ no processo.

Os métodos utilizados para determinação de $\mathrm{Hg}$, tanto por $\mathrm{CV}$ AFS quanto por ICP-MS, podem ser considerados semelhantes, sendo possível a aplicação de ambos em determinações rotineiras de $\mathrm{Hg}$.
Tabela 4. Concentração de $\mathrm{Hg}$ em amostra de fígado de tetrápodes marinhos in natura e liofilizadas analisadas por CV-AFS. Valores normalizados em base seca

\begin{tabular}{|c|c|c|}
\hline & In natura & Liofilizada \\
\hline Amostra & $\begin{array}{c}\text { Média } \pm \text { desvio padrão } \\
\left(\mathrm{mg} \mathrm{kg}^{-1}\right)\end{array}$ & $\begin{array}{c}\text { Média } \pm \text { desvio padrão } \\
\left(\mathrm{mg} \mathrm{kg}^{-1}\right)\end{array}$ \\
\hline PT 541 & $5,237 \pm 0,095$ & $5,019 \pm 0,165$ \\
\hline PT 543 & $0,577 \pm 0,048$ & $0,393 \pm 0,041$ \\
\hline PT 544 & $1,088 \pm 0,026$ & $0,777 \pm 0,011$ \\
\hline PT 545 & $0,532 \pm 0,058$ & $0,306 \pm 0,022$ \\
\hline PT 546 & $0,542 \pm 0,027$ & $0,361 \pm 0,029$ \\
\hline PT 547 & $0,756 \pm 0,109$ & $0,523 \pm 0,010$ \\
\hline PT 549 & $3,931 \pm 0,224$ & $2,288 \pm 0,052$ \\
\hline PT 550 & $0,687 \pm 0,040$ & $0,268 \pm 0,012$ \\
\hline PT 581 & $0,556 \pm 0,048$ & $0,289 \pm 0,018$ \\
\hline PT 582 & $1,121 \pm 0,021$ & $0,673 \pm 0,022$ \\
\hline PT 585 & $1,553 \pm 0,030$ & $1,069 \pm 0,020$ \\
\hline PT 586 & $1,264 \pm 0,036$ & $0,881 \pm 0,018$ \\
\hline PT 587 & $0,590 \pm 0,067$ & $0,390 \pm 0,028$ \\
\hline PT 588 & $0,622 \pm 0,123$ & $0,252 \pm 0,005$ \\
\hline PT 589 & $4,226 \pm 0,055$ & $3,219 \pm 0,092$ \\
\hline
\end{tabular}

Média e desvio padrão calculados para 3 leituras.

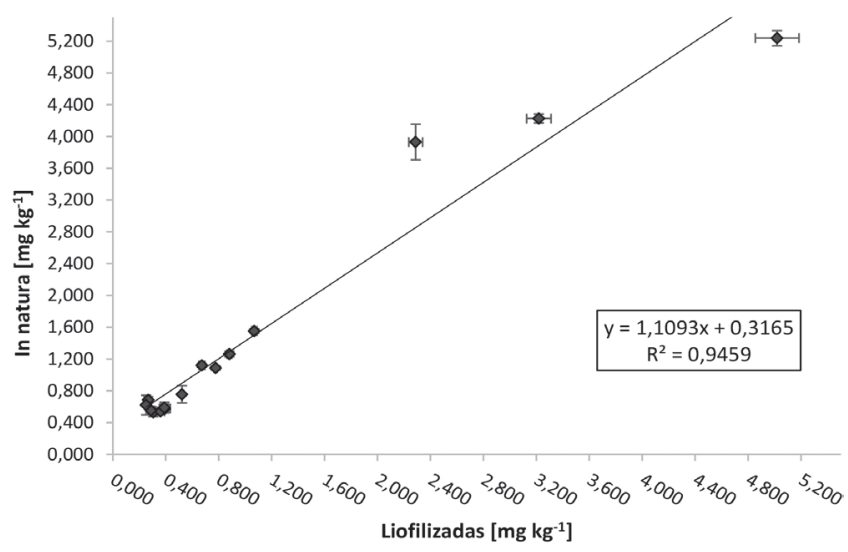

Figura 3. Correlação entre os valores de concentração de Hg encontrados in natura e liofilizadas por espectrometria de fluorescência atômica acoplada a geração de vapor frio (CV-AFS) em amostras de fígado de tetrápodes marinhos

\section{AGRADECIMENTOS}

Os autores agradecem ao CNPq, à CAPES pelas bolsas de estudo concedidas e à Petróleo Brasileiro S. A. - Petrobras pelos recursos finaceiros. Este trabalho é uma das consequências dos Projetos de Monitoramento de Praias da Bacia de Santos (PMP-BS fase 1 e fase 2) exigidos pelo IBAMA para o licenciamento ambiental da Atividade de Produção e Escoamento de Petróleo e Gás Natural no Pré-sal.

\section{REFERÊNCIAS}

1. de Azevedo, F. A.; Toxicologia do mercúrio, RIMA: São Paulo, 2003.

2. Bisinoti, M. C.; Jardim, W. F.; Quim. Nova 2004, 27, 593.

3. Kasper, D.; Forsberg, B. R.; Amaral, J. H. F.; Leitão, R. P.; Pie-Daniel, S. S.; Bastos, W. R. E.; Malm, O.; Envir. Sci. Techn. 2014, 48, 1032. 
4. Brito, B. C.; Forsberg, B. R.; Kasper, D.; Amaral, J. H. F.; de Vasconcelos, M. R. R.; de Sousa, O. P.; Cunha, F. A. G.; Bastos, W. R.; Hydrobiologia 2017, 790, 35.

5. Ibáñez-Palomino, C.; López-Sánchez, J. F.; Sahuquillo, A.; Anal. Chim. Acta 2012, 720, 9.

6. Loger, M.; Horvat, M.; Akagi, H.; Ando, T.; Tomiyasu, T.; Fajon, V.; Appl. Organometal. Chem. 2001, 15, 515.

7. Leermakers, M.; Baeyens, W.; Quevauviller, P. H.; Horvat, M.; Trends Anal. Chem. 2005, 24, 383.

8. Bloom, N.; Canadian Journal of Fisheries and Aquatic Sciences 1989, 46, 1131.

9. Sánchez-Rodas, D.; Corns, W. T.; Chen, B; Stockwell, P. B.; J. Anal. At. Spectrom. 2010, 25, 933.

10. Clevenger, W. L.; Smith B. W.; Winefordner, J. D.; Crit. Rev. Anal. Chem. 1997, 27, 1.

11. Takase, I.; Pereira, H. B.; Luna, A. S.; Grinberg, P.; Campos, R. C.; Quim. Nova 2002, 25, 1132.

12. Lamble, K. J.; Hill, S. J.; J. Anal. At. Spectrom. 1996, 11, 1099.

13. Tao, G.; Willie, S. N.; Sturgeon, R. E.; J. Anal. At. Spectrom. 1999, 14, 1929.

14. Tao, G.; Willie, S. N.; Sturgeon, R. E.; Analyst 1998, 123, 1215.

15. Park, C. J.; Hyunsu, D.; J. Anal. At. Spectrom. 2008, 23, 997.

16. Barcelos, J. S.; Relatório de estágio supervisionado, Universidade Federal de Santa Catarina, Brasil, 2015.

17. Menegário, A. A; Giné-Rosias, M. F.; Interferências em ICP-MS: Espectrometria de massas com fonte de plasma (ICP-MS), CENA: Piracicaba, 1999.

18. Thermo Scientific, AN-E0612 X Series ICP-MS, Clinical applications note 3: Determination of $\mathrm{Hg}$ in urine, Application Note, 2003.
19. Hintelmann, H.; Falter, R.; Ilgen, G.; Evans, D.; Fresenius, J.; Anal. Chem. 1997, 358, 363.

20. Rodriguez Martin-Doimeadios, R. C.; Monperrus, M.; Krupp, E.; Amouroux, D.; Donard, O. F. X.; Anal. Chem. 2003, 75, 3202.

21. Harrington, C. F.; Merson, S. A.; D’ Silva, T. M.; Anal. Chim. Acta 2004, 505, 247.

22. Zhu, X.; Alexandratos, S. D.; Microchem. J. 2007, 86, 37.

23. Gelaude, I.; Dams, R.; Resano, M.; Vanhaecke, F.; Moens, L.; Anal. Chem. 2002, 74, 3833.

24. Kenduzler, E.; Ates, M.; Arslan, Z.; McHenry, M.; Tchounwou, P. B.; Talanta 2012, 93, 404.

25. Wang, W.; Int. J. Pharm. 2000, 203, 1.

26. Schmidt, L.; Bizzi, C. A.; Duarte, F. A.; Dressler V. L.; Flores, E. M. M.; Microchem. J. 2013, 108, 53.

27. Ortiz, A. I. C.; Albarran, Y. M.; Rica, C. C.; J. Anal. At. Spectrom. 2002, 17, 1595.

28. Li, Y.; Yan, X.-P.; Dong, L.-M.; Wang, S.-W.; Yan, J.; Jiang, D.-Q.; J. Anal. At. Spectrom. 2005, 21, 94.

29. Gonzales, A.; Herrador, M.; Trends Anal. Chem. 2007, 26, 227.

30. Rice, E. W. Em Standard methods for the examination of water and wastewater; Rice, E. W., Bridgewater, L., eds.; American Water Works Association: Washington, DC, 2012.

31. Instituto Nacional de Metrologia, Qualidade e Tecnologia, Orientação sobre validação de métodos analíticos - DOQ-CGCRE-008 - Revisão 5, INMETRO: Rio de Janeiro, 2016.

32. Chen, J.; Determination of mercury in wastewater by inductively coupled plasma-mass spectrometry, Application Note, PerkinElmer, 2009.

33. Allibone, J.; Fatemian, E.; Walker, P. J.; J. Anal. At. Spectrom. 1999, 14, 235 . 